\title{
El certificado médico de defunción
}

\section{Medical certificate of death}

\author{
R. Bugarín González, B. Seoane Díaz. \\ Miembros de la Junta Directiva del Colegio Oficial de Médicos de A Coruña
}

La palabra "certificar" viene del latín certificare y quiere decir asegurar, afirmar, dar por cierto algo. Así, un certificado o certificación es un documento en el que se asegura la verdad, se da fe, de un hecho y, en nuestro contexto, hace referencia al estado de salud o de enfermedad de una persona. Por tanto, en su redacción deben constar únicamente datos objetivos, caso que no ocurre, por ejemplo, en los informes médicos en los que sí se pueden incluir argumentos valorativos 0 especulativos (aunque no arbitrarios sino debidamente razonados) como cuando se redactan los epígrafes referentes a "comentarios", "juicio clínico" o "impresión clínica".

En consecuencia, podemos decir que un certificado médico de defunción (CMD) es un documento, que sólo puede ser elaborado por un médico, en el que se da fe de la muerte de un ser humano.

En él, además, también se informa de la cadena de causas que produjo dicha muerte. Este proceso se determina o deduce con la misma sistemática que la mayor parte de los actos clínicos: a través de la historia clínica y de la exploración física. El diagnóstico de la causa o causas de la muerte es pues, con carácter general, un diagnóstico de presunción dado que no es necesaria la confirmación a través del gold standard que sería la autopsia. El diagnóstico de certeza se reserva para determinadas situaciones especiales como veremos en el desarrollo de este artículo.

Esto es importante tenerlo en cuenta ya que podría darse el caso de que cometamos un error diagnóstico (se da por supuesto que nos referimos a la causa, no al diagnóstico de la muerte en sî) lo cual no debería ser un motivo de preocupación si hemos sido rigurosos y sistemáticos en la elaboración de dicha hipótesis aunque posteriormente se demostrara errónea. En efecto, existe unanimidad en que es altamente improbable que se persiga judicialmente a algún médico que, de buena fe y con una actuación metódica, certifique como natural una muerte que no lo sea ${ }^{1,2}$.

\section{¿En qué impreso se certifica?}

El nuevo CMD entró en vigor en 2009 y consiste en un impreso que unifica el certificado médico y el boletín estadístico de defunción. Esta actualización fue realizada por la Organización Médica Colegial y el Instituto Nacional de Estadística tras consulta a los Ministerios de Justicia y de Sanidad ${ }^{1}$. Cumple con las recomendaciones de la Organización Mundial de la Salud³ .
El CMD tiene una doble función. Por un lado, documentar la muerte en el Registro Civil lo que hace posible la inhumación 0 incineración y, por otro, servir como soporte para las estadísticas de mortalidad. Por razones obvias, es de extraordinaria importancia la fiabilidad de dichos datos ya que tienen múltiples aplicaciones ${ }^{4}$. Así por ejemplo, permiten monitorizar los principales problemas de salud por los que muere la población, información de enorme utilidad para la planificación sanitaria. También se utilizan para realizar la vigilancia epidemiológica de alguna enfermedad. Asimismo, son imprescindibles para evaluar la calidad de la asistencia sanitaria de un servicio de salud. Por eso, es fácil entender que la incorrecta declaración de las causas de defunción repercutirá en las estadísticas de mortalidad ya que no reflejarán la realidad de las causas de muerte y esto tiene importantes implicaciones en diversos ámbitos sanitarios.

\section{¿Cuándo se debe cubrir el CMD?}

El médico asistencial debe cubrir el CMD, exclusivamente, en los casos de muerte natural y debe abstenerse ante la sospecha o posibilidad de un origen artificial. Por tanto, es imprescindible tener en cuenta estos conceptos ${ }^{5}$ :

Muerte natural. Aquella que tiene un origen interno. Es decir, la causa se originó "dentro del propio cuerpo". Hay que matizar que las muertes de etiología infecciosa también se consideran naturales aunque hay excepciones como podrían ser el tétanos secundario a una herida adquirida en un accidente de trabajo o el VIH contagiado tras una agresión con una jeringuilla.

Muerte violenta. La que tiene un origen exógeno. La causa que la motivó puede ser accidental o intencional y ésta, a su vez, suicida o criminal. Desde el punto de vista de la temporalidad, suele ser inmediata si bien, más raramente ocurre de forma retardada, es decir, diferida. Así por ejemplo, una fractura abierta debida a un accidente puede provocar, como secuela, una osteomielitis crónica y, bastante tiempo después, un shock séptico que conduzca a la muerte.

Muerte sospechosa de criminalidad. Es realmente un cajón de sastre. Se consideran aquí situaciones de diversa índole en las que, si bien externamente no hay signos de violencia, no hay datos que nos permitan conocer cuál ha sido la causa de la defunción. Dentro de este grupo se incluyen las muertes súbitas, las muertes sin asistencia médica y las muertes en el curso de un proceso clínico de evolución atípica. Por razones 
obvias, no podemos certificar en estos casos. Tampoco se debe hacerlo en muertes con posible responsabilidad médica (mala praxis) y en muertes de personas privadas de libertad ${ }^{6,7}$.

\section{¿Quién debe cubrir el CMD?}

Son ya clásicas las reticencias de los médicos a firmar los CMD. En ocasiones subyace el temor de que se le pase por alto un homicidio. Con frecuencia alegan, como motivo, que no conocen al paciente y que esta labor compete exclusivamente al médico de cabecera. Sin embargo, el Reglamento del Registro Civil ${ }^{8}$ dice textualmente en su artículo 274 que "el facultativo que haya asistido al difunto en su última enfermedad o cualquier otro que reconozca el cadáver enviará inmediatamente al Registro el parte de defunción". Por tanto, aun siendo reiterativos, vale la pena insistir en que, según este precepto, el CMD no tiene por qué firmarlo el médico que mejor conoce la historia clínica del paciente. Ni tan siquiera aquel que lo atendió en la enfermedad que le produjo la muerte sino que puede hacerlo cualquier médico que tenga la posibilidad de explorar el cadáver. La Declaración realizada por la Organización Médica Colegial sobre las Peculiaridades del certificado médico de defunción ${ }^{9}$ recomienda que dicho documento ha de extenderlo el médico que asistió al paciente durante el proceso que le condujo a la muerte, o el que estuvo presente en los últimos momentos, o el que lo atendió en su última enfermedad. Sólo en último caso, podrá redactarlo cualquier otro médico que haya reconocido el cadáver y pueda reconstruir fiablemente los mecanismos de muerte.

Por ello, a nuestro parecer, se puede decir que cualquier médico puede certificar una defunción siempre que se den todas y cada una de las siguientes tres premisas:

1. Tener la posibilidad de conocer los antecedentes patológicos del paciente.

2. Poder averiguar los pormenores del cuadro clínico que condujo a la muerte.

3. Poder realizar una exploración física del cadáver.

El conocimiento de los antecedentes del paciente se podrá obtener a través de informes clínicos o de la historia clínica electrónica.

La obtención de información del cuadro clínico que precedió a la muerte depende de cada caso en particular. Desde simplemente el interrogatorio a los allegados, cuando no se asistió en vida al paciente, 0 bien mediante datos averiguados a través de la anamnesis, exploración física e incluso de pruebas complementarias, cuando sí se tuvo la oportunidad de hacerlo. También puede ser útil recabar información a través de contacto telefónico con otros colegas, como por ejemplo con el médico de cabecera del fallecido.

La exploración física del cadáver deberá ser metódica y riguro- sa. Irá encaminada a buscar signos que nos permitan explicar la causa de la muerte (así por ejemplo una trombosis venosa profunda, en una pierna, nos orientaría como causa a una embolia pulmonar) y, sobre todo, para descartar la existencia de indicios que nos hagan pensar en un origen violento.

En este sentido, hay que tener en cuenta algunas particularidades. Los pacientes de edad avanzada, con frecuencia sufren cierto grado de púrpura senil y, si además están a tratamiento con corticoides, antiagregantes 0 anticoagulantes orales, son frecuentes las equimosis en los antebrazos sin que ello signifique un origen violento ${ }^{10}$. Tampoco debe confundirnos la herida en el cuero cabelludo producida por la caída tras una crisis epiléptica de un paciente que sufre un tumor cerebral. También es importante que el médico asistencial tenga habilidades para distinguir entre un hematoma y una lividez cadavérica ${ }^{5}$. Las livideces se producen al cesar la circulación lo que hace que la sangre se desplace a las partes declives debido a la fuerza de la gravedad. Tienden a la confluencia excepto en las zonas de apoyo. Aparecen a las 2-4 horas de la muerte, inicialmente se desvanecen a la vitropresión pero dejan de desaparecer con esta exploración cuando adquieren su máxima expresión en torno a las 12 horas. En la tabla 1 se recogen signos de alarma que nos pueden hacer sospechar que la muerte fue de origen violento.

Tabla 1. Signos de alarma

Excoriaciones alrededor de los labios, boca o cuello (pueden indicar
intento de sofocación)
Hematomas en la cara interna de los labios (intento de sofocación)
Petequias en la conjuntiva o en la esclera (homicidio por asfixia), no
confundir con la mancha de Sommer-Larcher (fenómeno cadavérico)
Ojos de mapache o hematoma en antifaz (fractura de la base del
cráneo)
Hematoma en el mastoides o signo de Battle (fractura de la base del
cráneo)
Hongo de espuma en las fosas nasales y en la boca (puede estar
en relación con una intoxicación por heroína o con una inmersión)
(también puede ocurrir en muertes naturales como el edema agudo
de pulmón)
Lesiones de defensa (hematomas en el borde interno de los
antebrazos y en las manos)

Fuente: modificado de Subirana (5)

Queda por tanto claro que existe una obligación legal de certificar y este deber no sólo compete al médico de cabecera sino que también se extiende a los médicos de urgencias, ya sea de las ambulancias medicalizadas del 061, como de Ios PAC o de los servicios de urgencias hospitalarios y, por supuesto, a los médicos de servicios hospitalarios cuando se trate de personas ingresadas. 
Además, también existe un deber ético. Muchas veces la inhibición del facultativo, fundamentalmente los fines de semana, alegando, como vimos, que no tuvo relación profesional previa con el fallecido 0 bien por un miedo infundado a cometer un error, genera que la muerte se convierta en judicial y sea obligatoria la autopsia. Esto provoca un sufrimiento innecesario a los familiares con una importante carga de angustia. Es prioritario que contribuyamos a la humanización y a la creación de un adecuado clima emocional en tales situaciones. Por eso, coincidimos con Monsó ${ }^{11}$ cuando dice que "si legalmente puedo, éticamente debo".

El Código de Deontología Médica de la Organización Médica Colegial $^{12}$, en el artículo 36.6 recoge que aunque el médico que haya tenido la mayor carga asistencial sobre el paciente es el que tiene la mayor responsabilidad ética de cumplimentar el certificado de defunción en todos sus apartados, no es deontológicamente aceptable rehuir el compromiso de certificarla cuando se produce si se ha presenciado la misma, se conoce al paciente o se tiene a disposición la historia clínica.

Cuando la defunción tiene lugar en un paciente ingresado en un centro sanitario o sociosanitario, salvo en raras excepciones, la certificación no suele plantear problemas. Las dificultades ocurren cuando el óbito se produce en el ámbito comunitario. Nos parece muy interesante el Acuerdo de Colaboración realizado entre el Departamento de Justicia y el Servicio Vasco de Salud ${ }^{13}$ ya que establece un protocolo que puede servir de gran ayuda para contribuir a disipar los "temores" de los profesionales. Consideramos que está muy bien sistematizado por lo que sería deseable que se realizaran iniciativas similares en otras Comunidades. En primer lugar delimita las competencias de cada institución: el servicio de salud se deberá encargar del CMD de todas las muertes excepto de las violentas o sospechosas de criminalidad que dependerán del Instituto Vasco de Medicina Legal. Así, ante un fallecimiento en el medio extrahospitalario, el médico asistencial (ya sea de PAC o del servicio de emergencias) debe realizar una inspección externa del cadáver para descartar lesiones compatibles con violencia, averiguar los antecedentes patológicos y los síntomas previos a la muerte y observar las circunstancias del entorno (ambiente sospechoso). Incluso recomienda que se tenga en cuenta la edad del fallecido. Si la impresión es que se trata de una muerte natural y existe la posibilidad de contactar con el médico de familia en las siguientes 24 horas, se le remitirá el informe asistencial de urgencias para que sea éste el que certifique la muerte. Si, en ese tiempo, no va a ser posible el contacto con el médico de familia por ser fin de semana o día festivo, el CMD lo cubrirá el propio médico que ha confirmado el éxitus. Incluso explicita que ante el fallecimiento de un paciente anciano en el que no haya indicios de muerte violenta o sospechosa de criminalidad y no existiesen antecedentes clínicos, se procederá a firmar el CMD indicando, si procede, senilidad. Por ello, tras la confirmación de la muerte por parte de un facultativo y excluida de forma razonable la muerte violenta o sospechosa de criminalidad, el cadáver podrá ser movilizado y trasladado a la funeraria.

Este Acuerdo, también establece indicaciones ante los pacientes fallecidos en servicios de urgencias hospitalarias. En las muertes naturales que fallecen en dicho servicio será el médico de urgencias el que certifique mientras que en los que ingresan ya cadáver, el CMD lo extenderá el médico que ha realizado la asistencia con la información recibida por familiares, por informes médicos previos o con la obtenida a través de la historia clínica informatizada.

En Madrid se elaboró un documento similar enfocado básicamente a la emisión de certificados de defunción por parte de Ios médicos del SUMMA112. En él se recoge una declaración que dice textualmente que, en la emisión de los certificados de defunción, no es exigible a dichos facultativos una infalibilidad absoluta en cuanto a la determinación de las causas del fallecimiento, siempre que se entiendan dichas causas como naturales ${ }^{14}$.

También creemos que es interesante la aportación que se hace desde la página web de la Consejería de Sanidad y Política Social de Murcia ${ }^{15}$. Una vez descartada la muerte violenta o sospechosa de criminalidad, puede ocurrir que el médico interviniente, con todos los datos disponibles, no sea capaz de llegar a una decisión adecuada para construir un criterio de causa de muerte que le sirva de esquema y así certificar. En cuyo caso recomienda dos alternativas: si se considera importante aclarar la duda clínica o epidemiológica debe solicitar una autopsia clínica al hospital de referencia. Por el contrario, si la duda no es relevante, el médico asistencial debe firmar la defunción como muerte natural utilizando como causa la más lógica dentro de los datos que haya podido obtener del fallecimiento.

Un último matiz, el artículo 274 del Reglamento del Registro Civil, al que antes aludimos ${ }^{8}$, incluye dentro de los datos identificativos del facultativo que suscribe, el número de colegiación por lo que entendernos que, según esta normativa, es imprescindible estar colegiado para poder cumplimentar un CMD.

\section{¿Cómo se debe cubrir el CMD?}

Nuestro modelo de certificación sigue las directrices de la Organización Mundial de la Salud³. Así, debe existir una secuencia lógica, fisiopatológica, y por tanto también temporal, de la concatenación de causas que condujeron a la muerte, de tal manera que la más cercana es la denominada causa inmediata que será consecuencia de una 0 unas causas intermedias y ésta o éstas, a su vez, estarán motivadas por una causa inicial o fundamental. Asimismo, pueden existir otros 
procesos que, si bien no están dentro de la cadena etiológica, forman parte de los antecedentes patológicos del fallecido y, por tanto, podrían haber contribuido de alguna manera al referido deceso. Por tanto, el médico certificador tiene que explicitar las causas concretas con los siguientes criterios:

- Causa inmediata de la defunción: enfermedad o estado patológico que produjo la muerte directamente. Es importante tener en cuenta que no se trata de indicar las maneras de morir sino la enfermedad, el traumatismo o la complicación causante del fallecimiento. Así el paro cardiaco es una manera de morir y por tanto debe evitarse su mención como causa.

- Causa o causas intermedias: son los estados morbosos que conducen a la causa inmediata y que son consecuencia de la causa fundamental. Lógicamente, no tienen por qué existir siempre.

- Causa inicial o fundamental: es la enfermedad o lesión que inició los hechos anteriormente mencionados que condujeron a la muerte. Se anotará únicamente una causa. A partir de aquí, el organismo responsable de la estadística, elegirá, teniendo en cuenta la Décima Revisión de la Clasificación Internacional de Enfermedades, la causa básica de la defunción y será ésta la que se tenga en cuenta a efectos estadísticos.

- Otros procesos: se trata de otros estados patológicos significativos que contribuyeron a la muerte, pero no están relacionados con la enfermedad o estado patológico que la produjo.

Al lado de cada uno de los estados patológicos inscritos en el CMD se reserva espacio para indicar el intervalo entre el presunto comienzo de la enfermedad y la fecha de la defunción. Este intervalo se anotará aunque sólo se conozca aproximadamente. En el impreso se facilitan cuatro unidades de tiempo (horas, días, meses y años) que son mutuamente excluyentes, es decir, hay que expresar el período en una única unidad de tiempo.

Una de las novedades del actual impreso de certificación es que incluye epígrafes para obtener información sobre si el óbito ha sido consecuencia diferida de un accidente de tráfico 0 laboral. Según Cicera y Segura ${ }^{1}$ esto puede generar al médico certificador dudas sobre la repercusión legal de su indicación ya que hay que tener en cuenta que toda defunción a consecuencia diferida de accidente es objeto del ámbito judicial.

En contraposición al anterior modelo de CMD, el impreso actual solamente recoge como forma de corroborar la identidad del cadáver, la identificación a través de un documento ya sea éste el DNI, el pasaporte o la tarjeta de residencia. Sin embargo, sigue vigente el artículo 274 del Reglamento del Registro Civil que admite otras dos fórmulas ya sea porque el fallecido es conocido de ciencia propia por el certificador 0 bien acreditada y, en este supuesto, se deben aportar, además, menciones de identidad de la persona que lo acredita, la cual también firmará el documento.
Tabla 2. Decálogo a tener en cuenta al certificar una defunción

1. Utilizar mayúsculas y preferentemente bolígrafo de tinta negra
2. Utilizar información real
3. Evitar abreviaturas, siglas y signos ortográficos, distintos de la coma
4. Describir la secuencia lógica de patologías
5. Certificar una única causa fundamental
6. Certificar enfermedades y síndromes sin ambigüedades
7. Evitar certificar entidades mal definidas como causa fundamental
8. Evitar certificar signos y síntomas
9. Evitar al máximo certificar mecanismos (diagnósticos) de muerte
10.Cumplimentar personalmente el Certificado Médico de Defunción

Fuente: www.certificaweb.es ${ }^{17}$

En determinados pacientes pluripatológicos puede ser complicado determinar cuál fue la causa fundamental. Como sólo se puede hacer constar una única causa, queda a criterio del clínico la elección de aquella que sea la más plausible.

Son numerosas las publicaciones que advierten del uso erróneo de "paro cardiaco" o "parada cardio-respiratoria" en los CMD. Raba y $\mathrm{col}^{16}$ consideran que posiblemente este hecho se deba a un conjunto de factores. Es más complicado conocer la causa inmediata de la muerte que la fundamental. Además, es menos importante la primera ya que la que se va a tener en cuenta, a efectos estadísticos, es la segunda. Esto hace que, con frecuencia, se utilice el término "paro" o "parada" como si fuera un comodín. Por otra parte, estos mismos autores señalan que también puede deberse a una deficiente formación en medicina legal que lleva a confundir las causas de la muerte con los mecanismos.

En ocasiones el facultativo debe decidir donde acota la temporalidad de la concatenación de causas. Así, un paciente de 78 años, fumador desde la adolescencia, diagnosticado en la edad media de una bronquitis crónica obstructiva que sufre una neumonía y muere, puede seleccionar como causa inicial o fundamental el tabaquismo 0 bien la enfermedad pulmonar de base y en ambos casos el CMD estará bien cumplimentado, independientemente de que el estadístico, posteriormente, seleccione como causa básica la bronquitis crónica obstructiva ya que las normas de codificación dan prioridad a la enfermedad respiratoria ${ }^{4}$.

También puede ocurrir que la implicación etiológica de un evento sobre otro no esté perfectamente establecida. A modo de ejemplo, un individuo, diabético desde hace bastantes años, que sufre una cardiopatía isquémica y muere como consecuencia de un infarto agudo de miocardio, la certificación podría partir, como causa inicial o fundamental, de la cardiopatía isquémica o de la diabetes ${ }^{4}$. 
Por último, es importante insistir en que en la cumplimentación del CMD se deben tener en cuenta una serie de recomendaciones para asegurar la calidad de los registros. Así, por ejemplo, para facilitar el reconocimiento óptico de caracteres, es recomendable que se escriba con letras mayúsculas y preferentemente con un bolígrafo de tinta negra. Por supuesto, se deben evitar símbolos y abreviaturas que puedan dificultar su lectura. También se aconseja evitar las interrogaciones sustituyéndolas con términos como por ejemplo "probable". Estas y otras recomendaciones se han recogido en forma de "Decálogo" (tabla 2) en la página web elaborada por el Ministerio de Sanidad, la Organización Médica Colegial, el Instituto Nacional de Estadística y algunas Comunidades Autónomas, entre ellas la gallega ${ }^{17}$.

\section{Bibliografía}

1. Cicera $L$, Segura A. Documentos médicos de la defunción actualizados: certificado médico de defunción y boletín estadístico de parto. Aten Primaria. 2010; 42: 431 437

2. Barbería E, Xifró A, Martín-Fumadó C, Arimany J. Consideraciones sobre la emisión del certificado médico de defunción. Aten Primaria. 2013; 45: 396-397.

3. Organización Mundial de la Salud. Certificación Médica de Causa de Defunción. Instrucciones para los médicos sobre el empleo del Modelo Internacional del Certificado Médico de Causa de Defunción. Ginebra: OMS; 1980. Disponible en: http:// whqlibdoc.who.int/publications/924356062X.pdf [Fecha de acceso: 19/10/2013]

4. Borrel C, Martos D. Cómo cumplimentar correctamente un boletín estadístico de defunción. FMC; 2002; 9: 383-392.

5. Subirana M. Cuándo sospechar que una muerte no es natural. FMC. 2012; 19 402-410.
6. Delgado G, Vila M. Consideraciones en la certificación de la muerte. Jano. 2009 1742: 37-39.

7. Salla RM. Certificados médicos de defunción. En: Semfyc. Guía de Actuación en Atención Primaria. $4^{\mathrm{a}}$ edición. Barcelona: Semfyc ediciones; 2011. p. 1510-1514.

8. Reglamento del Registro Civil. Decreto de 14 de noviembre de 1958, BOE del 11 de diciembre. (Actualización en el BOE de 19 de septiembre de 1986). Disponible en: www.mjusticia.gob.es [Fecha de acceso: 23/10/2013]

9. OMC. Declaración sobre las cualidades del certificado médico y sobre sus diferencias con los partes y los informes médicos. Peculiaridades del certificado médico de defunción; 2007. Disponible en: www.cgcom.es/sites/default/files/07_01_26_certificados_0.pdf [Fecha de acceso: 23/10/2013]

10. Casas J, Solé J. La púrpura en la visita de atención primaria. AMF. 2011; 7: 635 641.

11. Monsó C. ¿Firmo el certificado de defunción? Legalidad y ética. Aten Primaria. 2012; 44: 20-22

12. Organización Médica Colegial de España. Código de Deontología Médica. Guía de Ética Médica. Organización Médica Colegial de España; 2011.

13. Acuerdo de colaboración entre el Departamento de Justicia, Empleo y Seguridad Social a través de la dirección de relaciones con la Administración de Justicia y de Osakidetza a través de las direcciones de emergencias, de urgencias hospitalarias y extrahospitalarias de Osakidetza. Disponible en: http://www.murciasalud.es/recursos/ficheros/264565-ACUERD0.pdf. [Fecha de acceso: 24/10/2013]

14. Procedimiento de actuación para la emisión de certificados y partes de defunción en el Servicio de Urgencias de la Comunidad de Madrid. Disponible en: http://www. icomem.es/verDocumento.ashx?ld=831 [Fecha de acceso: 26/10/2013]

15. Consejería de Sanidad y Política Social de la Región de Murcia. Información jurídicolegal. 7. ¿Muerte judicial? Disponible en: http://www.murciasalud.es/pagina.php?id $=149757 \&$ idsec $=3714 \&$ expand $=1$ [Fecha de acceso: 25/10/2013]

16. Raba S, Villa M, Royano M, Ortiz I, Solar A, Díez A. Errores en la cumplimentación de los certificados médicos de defunción: la parada cardio-respiratoria como causa inmediata de muerte y sus consecuencias. Revista de la SEMG. 2004; 65: 349-353.

17. Ministerio de Sanidad y Consumo, Consejería de Sanidad de la Junta de Castilla y León, Departament de Salut de la Generalitat de Catalunya, Consellería de Sanidade da Xunta de Galicia, Consejería de Sanidad y Consumo de la Región de Murcia, Instituto de Salud del Gobierno Vasco, Organización Médico Colegial, Instituto Nacional de Estadística. Certifica. Disponible en: certificaweb.es [Fecha de acceso: 26/10/2013] 